\title{
Knowledge of medical doctors in Turkey about the relationship between periodontal disease and systemic health
}

\section{Zekeriya TAŞDEMIR Banu Arzu ALKAN}

Erciyes University, Faculty of Dentistry, Department of Periodontology, Kayseri, Turkey.

Declaration of Interests: The authors certify that they have no commercial or associative interest that represents a conflict of interest in connection with the manuscript.

Corresponding Author:

Zekeriya Taşdemir

E-mail: zekeriyatasdemir@hotmail.com

DOI: 10.1590/1807-3107BOR-2015.vol29.0055

Submitted: Oct 10,2014

Accepted for publication: Jan 02, 2015

Last revision: Mar 03, 2015

\begin{abstract}
Understanding the relationship between periodontal disease (PD) and systemic health (SH) is necessary for the accurate diagnosis and treatment of both. The aim of this study was to evaluate the knowledge of medical doctors in Turkey with regard to the association between PD and SH. This study was carried out using self-reported questionnaires that were sent to medical doctors who work at various universities and public and private hospitals in different cities in Turkey. The questionnaires consisted of questions about the demographic information of the medical doctors, as well as the knowledge of those doctors about the relationship between PD and SH. In total, 1,766 responses were received and $90.8 \%$ of the participants agreed that there was a relationship between PD and SH. Diabetes mellitus was the most frequent systemic disease (66.8\%) known to be related to $\mathrm{PD}$. Of the participants, 56.5\% of the medical doctors referred their patients to periodontists for different reasons. Gingival bleeding was the most frequent reason for patient referrals, with $44 \%$ of doctors giving such referrals. Doctors who worked in basic medical sciences were significantly less aware of the relationship between PD and $\mathrm{SH}$ than the doctors in other specialties. Although the vast majority of the medical doctors reported that they knew the relationship between $\mathrm{PD}$ and $\mathrm{SH}$, the findings of this study showed that this awareness was not supported by precise knowledge, and often failed to translate into appropriate clinical practice.
\end{abstract}

Keywords: Periodontal Diseases; Physicians; Knowledge; Attitude.

\section{Introduction}

Periodontal diseases (PDs), which are predominantly caused by Gram negative bacterial infections, are common conditions characterized by the destruction of periodontal tissues, including alveolar bone and connective tissue attachments. ${ }^{1}$

Dental biofilm, which elicits an inflammatory response in periodontal tissues, is a major factor in the etiology of PD. ${ }^{2}$ Systemic conditions can modulate the relationship between biofilm and the inflammatory response and may alter the progression and severity of the PD. ${ }^{3}$ This process may ultimately result in the loss of the diseased teeth. ${ }^{4} \mathrm{PD}$ is a common oral health problem worldwide, and variations in its prevalence may originate from differences in methodology and diagnostic criteria: ${ }^{5}$ whereas half of 
all American adults suffer from $P D^{6}$ this rate is much higher $\left(84.3 \%\right.$ of adults $35-44$ years old) in Turkey ${ }^{7}$ and other countries. ${ }^{8}$

The potential effects of systemic diseases on the periodontium have been reported in earlier studies, and a new branch of medicine defined as "medical periodontology" was proposed by Williams and Offenbacher, ${ }^{11}$ who pointed to the bidirectional relationship between periodontal diseases and systemic conditions such as diabetes mellitus, cardiovascular, cerebrovascular and respiratory diseases, preterm birth and low birth weight. Additionally, previous researches have shown that PD may impact the general health of an individual or change the course of systemic conditions. ${ }^{10,12}$ In the case of diabetes mellitus, alterations occur in the gingival crevicular fluid, collagen metabolism, host response, and microflora. Periodontal infections interfere with glycemic control via inflammatory mediators produced by inflamed periodontal tissues such as interleukin(IL)-1, IL-6, and tumor necrosis factor (TNF)-alpha, that can affect glucose and lipid metabolism. ${ }^{10,13}$ Additionally, periodontal infections are associated with cardiovascular and cerebrovascular diseases through the formation of atherosclerosis. Periodontal infections may play a role in atherosclerotic plaque formation, and periodontal pathogens can be isolated from these atherosclerotic plaques. If atherosclerotic plaques occur in brain arteries, they can cause stroke; when plaques occur in coronary arteries, they can lead to myocardial infarction. ${ }^{12,14}$ Preterm birth and low birth weight are notable health problems associated with PD, and PD related infections may lead to altered cytokine and hormone levels, which may lead to complications during pregnancy. ${ }^{15,16}$ The biological mechanisms involved in the relationship between PDs and respiratory diseases remain unclear, but aspiration of bacteria from the oropharynx into the lower respiratory tract may be to blame. ${ }^{17,18}$ In recent studies, the relationships between obesity, anxiety, stress, kidney disease, and periodontal health have also been investigated and there is a great deal of ongoing research in this area. ${ }^{3,19}$

As stated by the World Health Organization (WHO), oral diseases, including PDs, are a serious and essential part of the general health of the individual. ${ }^{20}$ For this reason, medical doctors should become familiar with and use this information, because they can alter the course of systemic diseases, as well as appropriately determine their treatment and prognosis. In light of this, we aimed to evaluate the knowledge of medical doctors in Turkey with regard to the association between systemic health (SH) and PD.

\section{Methodology Subjects}

This study was carried out using self-reported questionnaires that were sent to medical doctors who had e-mail addresses available through the websites of various universities and public and private hospitals in different cities in Turkey. The survey period was from January 2014 to April 2014. Questionnaires were mailed with a cover letter that informed the doctors about study objectives and participant confidentiality. It was emphasized that answering the questionnaire was optional. The survey was completed by a total of 1,766 (752 female and 1,014 male) participants who gave approval for this study.

\section{Instrument}

A self-reported questionnaire with 12 items was used, consisting of questions about the demographic information for these medical doctors, as well as their knowledge about the relationship between $\mathrm{PD}$ and $\mathrm{SH}$.

\section{Ethical Considerations}

The study protocol was approved by the Erciyes University Faculty of Medicine Ethics Committee (meeting date: January 10, 2014; decision no. 2014/28). Each subject electronically signed a consent form.

\section{Statistical analysis}

The obtained data were analyzed using SPSS version 15 for Windows (SPSS Inc., Chicago, USA). Data were described using frequency counts and percentages, and comparisons were made using the Chi-square analysis for different independent samples. The significance level was set as 0.05 .

\section{Results}

In total, 1,766 responses were received $(22.2 \%$ response rate). The demographic data for the medical doctors are shown in Table 1: males comprised 
$42.6 \%$, intern doctors made up $34.9 \%$, doctors who had $\leq 10$ years of practice constituted $40.8 \%$, and $57.4 \%$ of the doctors who were not interns worked at university hospitals.

Of the study participants, $1,537(87 \%)$ reported that they had knowledge about the symptoms of PD and 911 (59\%) of them knew that the primary clinical symptoms of PD is bleeding of the gingiva (Figure 1).

Of the doctors in this study, $(1,603) 90.8 \%$ of the participants agreed that there is a relationship between $\mathrm{PD}$ and $\mathrm{SH}$. For $28 \%$ of these doctors, this information was obtained from lessons or workshops (Figure 2). Diabetes mellitus was the most frequent systemic disease $(66.8 \%)$ known to be related to PD (Figure 3).

There was no statistically significant relationship between the years in practice and the awareness of a relationship between PD and SH ( $p>0.05)$. Among all of the specialties, more medical doctors who worked in the basic medical science division stated that there was not a relationship between $\mathrm{PD}$ and $\mathrm{SH}$ $(p<0.05)$. Taking the institution of the doctors into

Table 1. Demographic data for the $[1,766]$ medical doctors who responded to the questionnaire.

\begin{tabular}{lc}
\hline Characteristic & Number (\%) \\
\hline Age (years) & \\
$<25$ & $698(39.5 \%)$ \\
$25-45$ & $883(50 \%)$ \\
$>45$ & $185(10.5 \%)$ \\
Gender & \\
Male & $752(42.6 \%)$ \\
Female & $1,014(57.3 \%)$ \\
Years in practice & \\
$\leq 10$ & $720(40.8 \%)$ \\
$>10$ & $429(24.3 \%)$ \\
Intern & $617(34.9 \%)$ \\
Specialty & \\
Intern & $617(34.9 \%)$ \\
General practitioner & $214(12.1 \%)$ \\
Medical sciences & $403(22.8 \%)$ \\
Surgical sciences & $338(19.2 \%)$ \\
Basic medical science & $194(11 \%)$ \\
Institution (interns were excluded from this category) \\
Public hospital & $341(29.7 \%)$ \\
Private hospital & $148(12.9 \%)$ \\
University hospital & $660(57.4 \%)$ \\
\hline
\end{tabular}

consideration, a greater percentage of doctors in public hospitals reported that there was not a relationship between $\mathrm{PD}$ and $\mathrm{SH}$ as compared with interns and doctors in university hospitals $(\mathrm{p}<0.05)$ (Table 2$)$.

Of the participants, $56.5 \%$ of the medical doctors referred their patients to periodontists for different reasons. Gingival bleeding was the most frequent reason for patient referrals, with a percentage of $44 \%$, and general practitioners most frequently referred their patients when compared with doctors with other specialties $(\mathrm{p}<0.05)$ (Table 2$)$.

\section{Discussion}

The possible relationship between PD and SH has already been investigated in recent studies. ${ }^{910,12,14}$ An understanding of this connection is vital for the

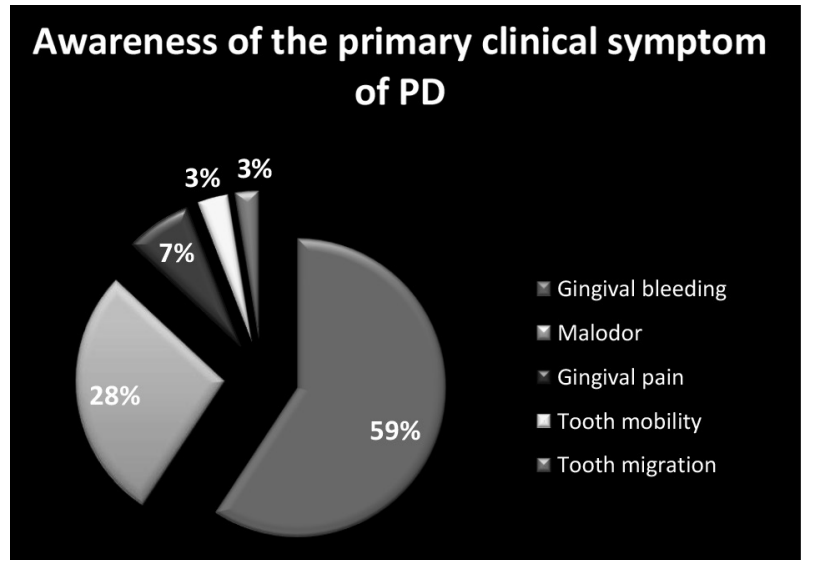

Figure 1. Awareness of the primary clinical symptom of PD.

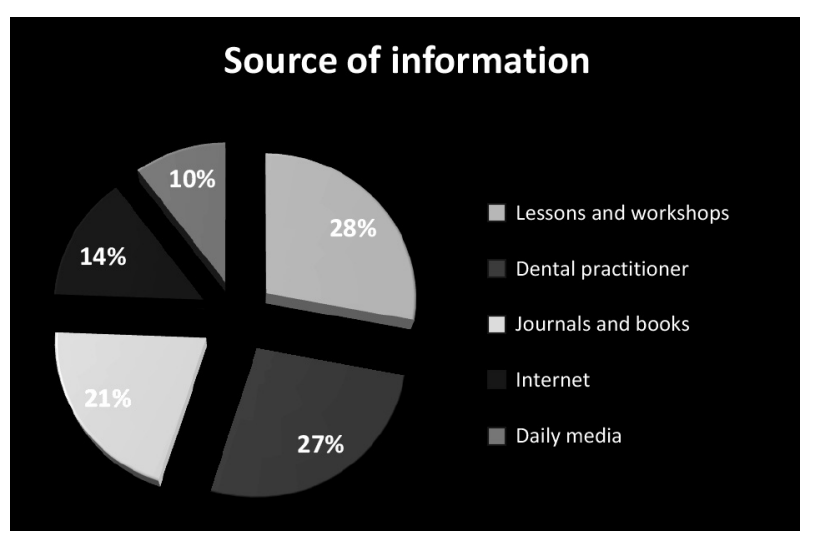

Figure 2. Source of information regarding the relationship between PD and systemic disease/conditions. 


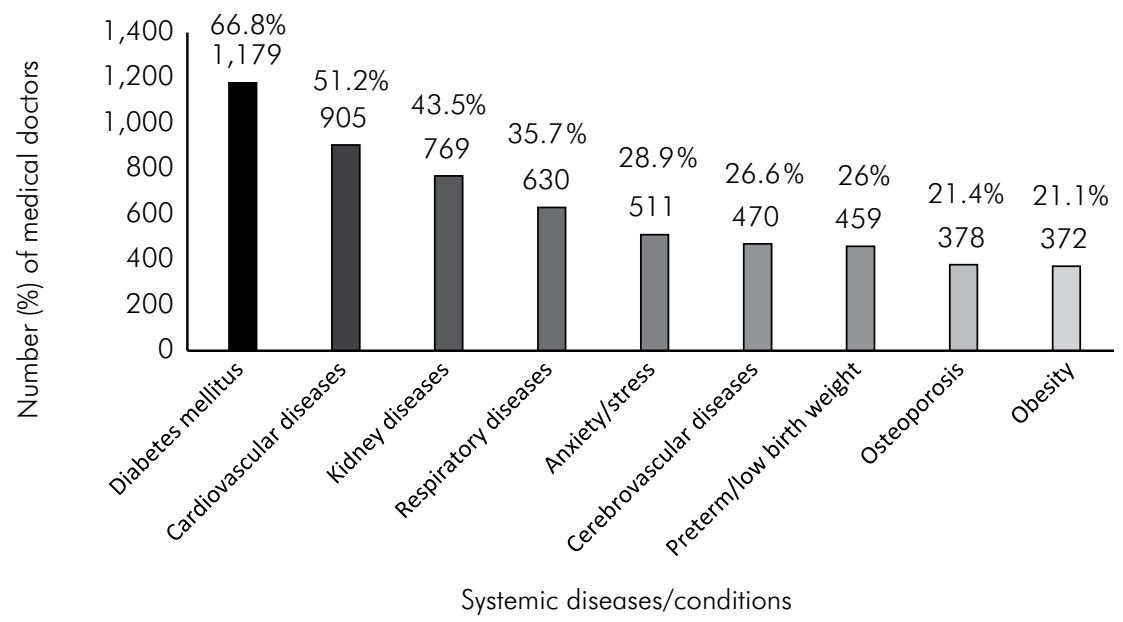

Figure 3. Knowledge of participants about the relationship between PD and systemic diseases/conditions.

proper diagnosis, treatment and maintenance of both periodontal and systemic diseases. Clinical observations give the impression that medical doctors are less interested in the relationship between PD and SH when compared with periodontists. ${ }^{21,22,23,24,25,26}$ Of the few studies that have investigated the knowledge of medical doctors about the relationship between $\mathrm{PD}$ and $\mathrm{SH}$. Most have focused on the relationship between a certain specific systemic disease/status and a certain specialty. ${ }^{24,25,26}$ Our study differs from these earlier ones in that we took into consideration different specialties among the doctors who participated and various systemic diseases/conditions.

Eighty-seven percent of medical doctors in this study reported that they knew the symptoms of PD. This rate ranges from $94.6 \%$ to $98 \%$ in other studies. ${ }^{25,26}$ This difference may by related to the different types of questions and expected responses (true/false vs. true/false) used in the different studies. We checked the knowledge of the respondents on PD symptoms with a question that asked what the primary symptom of $\mathrm{Pd}$ is. It is a striking finding that $41 \%$ of the medical doctors did not know the primary symptom of PD.

A few studies have investigated the awareness among medical doctors of an association between PD and SH. ${ }^{21,22,23,24,25}$ Pregnancy, diabetes mellitus, coronary heart disease, respiratory disease, and kidney disease are all diseases that have been investigated in this regard.
Table 2. Number of responding doctors who supported or denied the existence of a relationship between PD and SD.

\begin{tabular}{llll}
\hline & \multicolumn{3}{c}{ Relationship between PD and SD } \\
\cline { 2 - 4 } & Yes & No & p-value \\
\hline Years in practice & & & $<0.05$ \\
Intern & $652^{a}$ & $68^{a}$ & \\
$\leq 10$ & $383^{a}$ & $46^{a}$ & \\
$>10$ & $568^{a}$ & $49^{a}$ & \\
Specialty & & & $<0.05$ \\
Intern & $568^{a}$ & $49^{a}$ & \\
Basic medical science & $154^{b}$ & $40^{b}$ & \\
Surgical sciences & $318^{a}$ & $20^{a}$ & \\
Medical sciences & $370^{a}$ & $33^{a}$ & \\
General practitioner & $193^{a}$ & $21^{a}$ & \\
Institution & & & $<0.05$ \\
Intern & $568^{a}$ & $49^{a}$ & \\
University hospital & $612^{a}$ & $48^{a}$ & \\
Public hospital & $291^{b}$ & $50^{b}$ & \\
Specialty hospital & $132^{\mathrm{a}}$ & $16^{\mathrm{ab}}$ & \\
\hline
\end{tabular}

Different superscript letters represent significant differences (vertically within a category).

In our study, $90 \%$ of the medical doctors stated that a possible relationship between PD and $\mathrm{SH}$ exists. It is difficult to interpret this finding with the findings of Quijano et al. ${ }^{22}$ in which interns constituted almost the entire study population and answers to this question were stratified according to the level of knowledge. ${ }^{22}$ Medical doctors got the information regarding the relationship $\mathrm{PD}$ and $\mathrm{SH}$, primarily from lessons/workshops,dental practitioners and 
scientific or medical journals. This finding can be compared with the findings of Al-Habashneh et al. ${ }^{27}$ which showed that the responders obtained the same information, primarily from magazines. The different findings in the two studies may be explained by their different study populations: our responders were mostly interns and university doctors, whereas the population in the Al-Habashneh et al. ${ }^{27}$ study focused on general practitioners.

Although the rate of knowledge about the association between PD and diabetes mellitus in our study was $66.8 \%$, it was $42.2 \%$, and $97 \%$ in the studies of Al-Khabbaz et al. ${ }^{21}$ (general practitioner) and Quinjano et al. ${ }^{22}$ (interns), respectively. Lower knowledge rates about the association between PD and other systemic diseases in our study as compared with those in earlier studies $^{19,23}$ (kidney: $43.5 \%$ vs. $74.2 \%$, cardiovascular disease: $51.2 \%$ vs. $89 \%-95.1 \%$ ) was another prominent finding. This may be explained by the heterogenic distribution (interns, general practitioners, specialists) of the respondents in our study population.

No significant relationship between the number of years in practice and the awareness of a relationship between PD and SH existed in our study. This finding is not in accordance with the findings of Rocha et al. ${ }^{28}$ who reported that the length of time in practice among obstetricians was positively correlated with the frequency of recommendation for dental examinations during pregnancy. This discrepancy may be explained by differences in study populations and years in practice.

For doctors in this study thier specialty and institution showed significant relationships with the awareness of the relationship between PD and SH. Doctors who worked in basic medical sciences were less aware of this relationships than the doctors in other specialties. This may not be a surprising finding, as doctors working in basic medical sciences do not meet with patients in clinical practice. As expected, doctors working in public hospitals were less aware of the relationship between $\mathrm{PD}$ and $\mathrm{SH}$ when compared with interns and doctors working in university hospitals. The majority of the doctors working in public hospitals were general practitioners (62.7\%), who may be less likely to attend congresses/symposiums where such current issues are discussed.
Previous studies have investigated the referral rates of patients by medical doctors regarding the relationship between $\mathrm{PD}$ and SD. The referral rate $(56.5 \%)$ in the present study may be considered similar when compared with the findings of Al-Habashneh et al. ${ }^{27}$ and Owen et al. ${ }^{24}$, with rates of $49.7 \%$ and $48 \%$, respectively. Our findings underscore the need for promoting collaboration between dental and medical doctors to improve patient outcomes with respect to PD and associated diseases and conditions. For this purpose, a better education program of education about the relationship between $\mathrm{PD}$ and $\mathrm{SH}$ during undergraduate and postgraduate education and training is mandatory. In the presence of systemic diseases/conditions related to inflammation, the relationship between PD and SH should not be overlooked by medical doctors. Patients could better be treated in a multidisciplinary fashion upon consultation with a dental practitioner.

The inclusion of a large number of doctors from different institutions and specialties and the investigation of the relationship between various systemic diseases/conditions and PD rather than between a specific systemic disease/condition and PD are the strengths of our study compared with the other studies in the literature. ${ }^{21,22,23,24,25,26,27,28}$ The low reponse rate $(22.2 \%)$ which is typical of other mailed questionnaire-based studies of medical practitioners, ${ }^{29,30}$ could be considered a limitation of the present study but one point should be born in mind that, no strategy like sending reminder and second mail for increasing responses to the questionnaires was used. Another limitation was that the self-reported questionnaire might lead to bias in the answers given by the medical doctors. However, we attempted to minimize this bias by sequencing the questions and arranging the scope of the questionnaire.

\section{Conclusion}

Although the vast majority of the medical doctors reported that they knew the relationship between periodontal disease and systemic health, the findings of this study showed that this awareness was not supported by precise knowledge, and failed to lead to appropriate clinical practice. Interdisciplinary 
medical and dental training will likely be the solution to this problem.

\section{References}

1. Mane A, Karmarkar A, Bharadwaj R. Anaerobic bacteria in subjects with chronic periodontitis and in periodontal health. J Oral Health Comm Dent. 2009 Sep;3(3):49-51.

2. Kesic L, Milasin J, Igic M, Obradovic R. Microbial etiology of periodontal disease- mini review. Facta Univ Ser Med Biol. 2008;15(1):1-6.

3. Oppermann RV, Weidlich P, Musskopf ML. Periodontal disease and systemic complications. Braz Oral Res. 2012;26Suppl 1:39-47.

4. Gur A, Majra J. Awareness regarding the systemic effects of periodontal disease among medical interns in India. J Glob Infect Dis. 2011 Apr;3(2):123-7.

5. Oppermann RV. An overview of the epidemiology of periodontal diseases in Latin America. Braz Oral Res. 2007;21 Suppl 1:8-15.

6. Eke PI, Dye BA, Wei L, Thornton-Evans GO, Genco RJ. Prevelance of periodontitis in adults in the United States: 2009 and 2010. J Dent Res. 2012 Oct;91(10):914-20.

7. Gokalp S, Dogan BG, Tekcicek M, Berberoglu A, Unluer S. The oral health profile of adults and elderly, Turkey-2004. Clin Dent Res. 2007;31(4):11-8. Turkish

8. Susin C, Dalla Vecchia CF, Oppermann RV, Haugejorden $\mathrm{O}$, Albandar JM. Periodontal attachment loss in an urban population of Brazilian adults: effect of demographic, behavioral, and environmental risk indicators. J Periodontol. 2004 Jul;75(7):1033-41.

9. Kinane DG MG. Periodontal manifestations of systemic disease: Review. Aust Dent J 2001;46:2-12.

10. Kim J, Amar S. Periodontal disease and systemic conditions: a bidirectional relationship. Odontology. 2006 Sep;94(1):10-21.

11. Williams RC, Offenbacher S. Periodontal medicine: the emergence of a new branch of periodontology. Periodontology 2000. 2000 Jun;23:9-12.

12. Amar S, Han X. The impact of periodontal infection on systemic diseases. Med Sci Monit. 2003 Dec;9(12):RA291-9.

13. Preshaw PM, Alba AL, Herrera D, Jepsen S, Konstantinidis A, Makrilakis K, et al. Periodontitis and diabetes: a two way relationship. Diabetologia. 2012 Jan;55(1):21-31.

14. Khader YS, Albashaireh ZS, Alomari MA. Periodontal diseases and the risk of coronary heart and cerebrovascular diseases: a meta-analysis. J Periodontol. 2004 Aug;75(8):1046-53.

15. Sitholimela CS, Shangase LS. The association between periodontitis and pre-term birth and/or low birth weight: a literature review. SADJ. 2013 May;68(4):162-6.

16. Hasegawa-Nakamura K, Tateishi F, Nakamura T, Nakajima Y, Kawamata K, Douchi, et al. The possible mechanism of preterm birth associated with periodontopathic

\section{Acknowledgements}

This study was self funded by the authors.

Porphyromonas gingivalis. J Periodontal Res. 2011 Aug;46(4):497-504.

17. Bansal M, Khatri M, Taneja V. Potential role of periodontal infection in respiratory diseases - a review. J Med Life. 2013 Sep 15;6(3):244-8.

18. Scannapieco FA, Mylotte JM. Relationships between periodontal disease and bacterial pneumonia. J Periodontol. 1996 Oct;67(10 Suppl):1114-22.

19. Gulati M, Anand V, Jain N, Anand B, Bahuguna R, Govila $\mathrm{V}$, et al. Essentials of periodontal medicine in preventive medicine. Int J Prev Med. 2013 Sep;4(9):988-94.

20. World Health Organization. The Liverpool Declaration: Promoting Oral Health in the 21st Century. A call for action. Geneva: WHO; 2005. 2 p.

21. Al-Khabbaz AK, Al-Shammari KF, Al-Saleh NA. Knowledge about the association between periodontal diseases and diabetes mellitus: contrasting dentists and physicians. J Periodontol. 2011 Mar;82(3):360-6.

22. Quijano A, Shah AJ, Schwarcz AI, Lalla E, Ostfeld RJ. Knowledge and orientations of internal medicine trainees toward periodontal disease. J Periodontol. 2010 Mar;81(3):359-63.

23. Rabiei S, Mohebbi SZ, Patja K, Virtanen JI. Physicians' knowledge of and adherence to improving oral health. BMC Public Health. 2012 Oct 9;12:855.

24. Owens JB, Wilder RS, Southerland JH, Buse JB, Malone RM. North Carolina internists' and endocrinologists' knowledge, opinions, and behaviors regarding periodontal disease and diabetes: need and opportunity for interprofessional education. J Dent Educ. 2011 Mar;75(3):329-38.

25. Asa'ad F, Al-Maflehi N, Alelyan B, Asaad L, Alrumaih W, Alasaad F, et al. Knowledge and orientations of medical interns toward periodontal disease in Saudi Arabia. Saudi J Oral Sci. 2014 Jul;1(2):98-104.

26. Bastos JA, Vilela EM, Henrique MN, Daibert PC, Fernandes LFM, Paula DA, et al. Assessment of knowledge toward periodontal disease among a sample of nephrologists and nurses who work with chronic kidney disease not yet on dialysis. J Bras Nefrol. 2011 Dec;33(4):431-5.

27. Al-Habashneh R, Aljundi SH, Alwaeli HA. Survey of medical doctors' attitudes and knowledge of the association between oral health and pregnancy outcomes. Int J Dent Hyg. 2008 Aug;6(3):214-20.

28. Rocha JM, Chaves VR, Urbanetz AA, Baldissera RS, Rosing CK. Obstetricians' knowledge of periodontal disease as a potential risk factor for preterm delivery and low birth weight. Braz Oral Res. 2011 May-Jun;25(3):248-54. 
29. Rogers A, Furyk J, Banks C, Chu K. Diagnosis of subarachnoid haemorrhage: a survey of Australasian emergency physicians and trainees. Emerg Med Australas. 2014 Oct;26(5):468-73.
30. Berthelot S, Lang E, Quan H, Stelfox H. What are emergency-sensitive conditions? A survey of Canadian emergency physicians and nurses. CJEM. 2014 Aug;16:37-43.

\section{Questionnaire.}

1. Gender

$\square$ Female

Male

2. Age

$\square<25$ years

25-45 years

$>45$ years

3. When did you graduate from medical faculty?

$\square \leq 10$ years ago

$\square>10$ years

$\square$ Intern doctor (not graduated)

4. What is your institution? (Intern doctors must not answer this question)

Public hospital

Private hospital

University hospital

5. What is your medical specialty?

Basic medical sciences

Surgical sciences

Medical sciences

General practitioner

Intern doctor

6. Have you ever referred your patient to a dentist?

$\square$ Yes

No

If your answer is "Yes" please answer the next question.

7. What was the most frequent reason for referral?

Presence of intra oral lesion

Malodor

Gingival bleeding

Oral findings of systemic disease

Knowledge on relationship between periodontal disease and systemic disease

Before any medical intervention to minimize oral microbial burden 
Knowledge of medical doctors in Turkey about the relationship between periodontal disease and systemic health

\section{Continuation}

8. Do you know the symptoms of periodontal disease?

$\square$ Yes

$\square$ No

If your answer is "Yes" please answer the next question.

9. What is the primary clinical symptom of periodontal disease?

$\square$ Malodor

Tooth mobility

Tooth migration

$\square$ Gingival bleeding

$\square$ Gingival pain

10. Is there any relationship between periodontal disease and systemic health?

$\square$ Yes

$\square$ No

If your answer is "Yes" please answer the question 11 and 12.

11. Which systemic disease/conditions are related with periodontal disease?

( You are free to mark more than one choice)

Osteoporosis

Obesity

Anxiety/stress

Respiratory diseases

Cardiovascular diseases

Diabetes mellitus

Pretern/low birth weight

Kidney disease

Cerebrovascular diseases

12. Where did you get this information?

Dental practitioner

Internet

Daily media

Journals and books

Lessons and workshops 